\title{
Prevalence of Dementia in Older Latinos: The Influence of Type 2 Diabetes Mellitus, Stroke and Genetic Factors
}

\author{
Mary N. Haan, MPH, DrPH, *Dan M. Mungas, PhD, ${ }^{\dagger}$ Hector M. Gonzalez, PhD, * \\ Teresa A. Ortiz, BSN, ${ }^{+}$Ananth Acharya, MD, ${ }^{+}$and William J. Jagust, $\mathrm{MD}^{\dagger}$
}

OBJECTIVES: To estimate dementia prevalence in older Mexican Americans, determine the distribution of dementia by etiology, and evaluate the contribution of type 2 diabetes mellitus, stroke, and apolipoprotein E (ApoE) genotype to dementia.

DESIGN: Analysis of baseline data from an epidemiological cohort study.

SETTING: Sacramento Valley, California.

PARTICIPANTS: One thousand seven hundred eightynine Latinos aged 60 and older residing in targeted census tracts during 1998-99.

MEASUREMENTS: Each subject was interviewed and screened for dementia and cardiovascular risk factors and diseases. Fasting blood samples were drawn for glucose, insulin, and lipids. Buccal cells were obtained for genetic analysis of ApoE. A three-stage process of screening was used to diagnose dementia, including cognitive testing, a clinical examination, and imaging to determine etiology. Presence of dementia was established according to $\mathrm{Na}$ tional Institute of Neurological Disorders and Stroke/ Alzheimers and Related Disorders Association criteria and California Alzheimer's Disease Diagnostic and Treatment Criteria.

RESULTS: Overall dementia prevalence was $4.8 \%$. Prevalence in those aged 85 and older was $31 \%$. Education and Anglo cultural orientation was negatively associated with dementia risk. Risk of dementia was nearly eight times higher in those with both type 2 diabetes mellitus and stroke. Forty-three percent of dementia was attributable to type 2 diabetes mellitus, stroke, or a combination of the two. ApoE allele frequency was E2 5.9\%, E3 $90.1 \%$, and E4 4\%. Those with any E4 and 4-4 combinations had a higher risk for dementia than those with the E3-3 combination.

From the *Department of Epidemiology, University of Michigan School of Public Health, Ann Arbor, Michigan; and ${ }^{\dagger}$ Department of Neurology, University of California at Davis School of Medicine, Davis, California. Supported by NIA Grants AG 12975, AG 10129, and AG10220.

Address correspondence to Mary N. Haan, MPH, DrPH, University of Michigan, School of Public Health, Department of Epidemiology, 109 S. Observatory Street, Room 3005, Ann Arbor, MI 48109. E-mail: mnhaan@umich.edu
CONCLUSIONS: Dementia prevalence in this ethnic group is similar to that reported in Canadian and European studies but lower than in Caribbean-Hispanics residing in the United States. The etiological fraction of dementia attributable to type 2 diabetes mellitus and stroke is substantial and points toward the need for intervention research and treatment with the goal of reducing neurological sequelae in groups with high prevalence of type 2 diabetes mellitus. The allele frequency of ApoE was similar to that in other published studies on Mexican Americans. The low frequency of the E4 allele may contribute to the difference in etiology of dementia in older Mexican Americans and older people of European background. Dementia in this ethnic group may be related to preventable causes, with a smaller genetic component than in Europeans. J Am Geriatr Soc 51:169-177, 2003.

Key words: dementia; diabetes mellitus; genetics; Mexican; stroke

$I^{n}$ ncreased survival to older ages of the U.S. and world population has focused new attention on the causes and consequences of late-life cognitive impairment and dementia. There has been a rapid increase in the United States of older ethnic minority populations through a combination of immigration and improved survival in ethnic communities. This change has stimulated cross-cultural research into dementia that can provide insights about the relative occurrence of disease and help to identify new risk factors. Immigration is a major life event, and the process of adopting new cultural values and practices often changes exposure to risk factors and the course of many diseases, including dementia. ${ }^{1}$

There is evidence that prevalence and patterns of dementia in Latino subgroups may differ from those in European Americans. ${ }^{2,3} \mathrm{~A}$ prevalence study of dementia in three ethnic groups in New York City found that Caribbean Latinos were at significantly higher risk for dementia than European Americans. ${ }^{3}$ Accurate identification of dementia may be influenced by linguistic, educational, or cultural biases in standard testing instruments. ${ }^{4-6}$ Dementia may be 
overestimated in ethnic minorities who have limited education or whose primary language is not English. Lower education is consistently associated with worse health status on a number of outcomes, including dementia. Determining whether the effects of education on dementia are due to the worse health generally associated with lower socioeconomic status is difficult. Differences in population distributions of the apolipoprotein $\mathrm{E}$ (ApoE) genotype may also influence ethnic differences in risk of dementia.

This paper presents prevalence estimates for dementia for the Sacramento Area Latino Study on Aging (SALSA). The contributions of cerebrovascular disease, type 2 diabetes mellitus, and the ApoE genotype to this risk were examined. The SALSA is an ongoing cohort study of 1,789 Latinos aged 60 and older in 1998-99 residing in rural and urban areas of the Sacramento Valley surrounding and including the Sacramento Metropolitan Statistical Area.

\section{METHODS}

\section{Study Population and Recruitment}

Study participants were residents of the Sacramento Metropolitan Statistical Area and surrounding suburban and rural counties in California. An eligible person was aged 60 or older in 1998 and self-designated as Latino. The first stage of the sampling involved identifying 1990 Census tracts in five contiguous counties and characterizing them by the percentage of eligible residents (aged $\geq 60$ and Latino). These tracts were ranked in order of percentage eligible, and all tracts in which the percentage eligible was at least $5 \%$ were selected for the target population. Because the recruitment occurred 8 years after the 1990 Census, a phone and address list was purchased of people aged 60 and over with Latino surnames in the target area. This list was used to identify census tract areas where there might have been a change in the eligible population since 1990 and to contact individuals in the selected census tracts. Participants were contacted in three stages: by mail, by phone, and, finally, by door-to-door neighborhood enumeration. Up to 10 attempts to contact the participant by telephone were allowed and up to five attempts at the household. Participants who contacted the study themselves were screened for eligibility, residence in a target census tract, and presence on the sampling list. The overall response rate in those contacted was $85 \%$. Approximately $22 \%$ of the total eligible population of Sacramento County was recruited. ${ }^{7}$ All eligible household residents could be included in the study, and 790 participants were living with at least one other study participant. At baseline (1998-99), 1,789 people were enrolled in the study. The methods of enumeration and recruitment process have been published elsewhere, along with a comparison of the resulting sample to the Census 2000 Dress Rehearsal data on Hispanics for the target area. ${ }^{8}$ The sample was highly representative of older Hispanics residing in the target area. More than $89 \%$ of eligible households that were enumerated had at least one person who participated in the study. Participation in the cognitive screening was not an eligibility criterion for inclusion in the overall study.

\section{Data Collection}

All field staff was bilingual in Spanish and English, and participants were interviewed in their language of choice.
All data collection was done at the participants' homes. In a 2-hour interview, each participant answered questions about lifestyle factors, depressive symptoms, acculturation, and medical diagnoses. Blood pressure and ankle:arm blood pressure were measured, and fasting blood was drawn for measurement of lipids, antioxidants, glucose, and insulin. Buccal swabs were taken to obtain deoxyribonucleic acid (DNA) for ApoE analysis. Participants completed physical performance tests, including a tandem stand and walk, multiple chair stands, and a timed walk. If the participants were unable to respond verbally, had a score below 40 on the Modified Mini-Mental State Examination (3MSE), or the caregiver indicated that the participant was unable to respond but still wanted him or her to be tested, a proxy interview was done that included only questions that a third party could appropriately answer. In addition, the caregivers were assessed for their frequency of contact with the participants; nearly all were spousal caregivers or otherwise living with the participant.

Cultural orientation was measured using the Acculturation Rating Scale for Mexican Americans. ${ }^{9}$ This instrument asks subjects about which language they prefer, whether they prefer Spanish or English media, and about contacts with country of origin and with people of Latino versus Anglo background. The scale was formed so that the least affiliation with Anglo culture was scored as 0, and the items were summed to form a scale (0-37). Cultural orientation scores for those who were missing values $(74 / 1,789)$ were estimated from means for the same age, sex, birthplace, and education. Type 2 diabetes mellitus was ascertained by determining use of a diabetic medication (obtained by medicine chest inventory at the interview), self-report of a physicians' diagnosis, or fasting glucose of $126 \mathrm{mg} / \mathrm{dL}$ or greater. Stroke was ascertained using self-report of physician's diagnosis. Education was measured as years of formal schooling. Activities of daily living (ADLs) and instrumental activities of daily living (IADLs) were measured using a standard Likert-type scale. ${ }^{10}$ Two cognitive screening tests were used to determine the need for further neuropsychological evaluation. These were the $3 \mathrm{MSE}^{11}$ and the Spanish and English Verbal Learning Test (SEVLT). ${ }^{12}$ The $3 \mathrm{MSE}$ is a measure of global cognitive functioning that is strongly associated with dementia. The delayed recall trial (DelRec) from the SEVLT test was used with the 3MSE to screen for cognitive impairment. Scores on these two tests were statistically adjusted for effects of age and education of test administration using previously reported methods, ${ }^{12-14}$ The intent of this adjustment was to reduce the false-positive rate due to education and age effects on cognitive test scores. Participants were referred for formal neuropsychological evaluation if adjusted screening scores fell below the 20th percentile on either adjusted test $(3 \mathrm{MSE}<84$ or DelRec < 7). The 20th percentiles for the unadjusted tests were scores less than 6 on the DelRec and less than 79 on the 3MSE. All participants whose scores met the adjusted criteria were referred for a neuropsychological test battery. In addition, a $20 \%$ subsample (tagged) of the entire sample was referred for neuropsychological testing. Random systematic sampling was used for creating this subsample. Every fifth person recruited and screened was referred to neuropsychological testing without regard to his or her 
screening test results. The percentage of subjects who met criteria for impairment on the screening tests did not significantly differ $(P \leq .08)$ according to initial contact method (phone, home visit, referral).

The neuropsychological test battery (Spanish English Neuropsychological Assessment Scales (SENAS))14 was constructed with five scales to provide psychometrically matched English- and Spanish-language measures of abilities that are relevant to the clinical assessment of cognitive functioning in older persons. These scales included verbal and nonverbal measures of semantic memory, verbal attention span, verbal abstraction, and visual-perceptual ability. Results from a previous study ${ }^{15}$ show that these scales are equally sensitive to cognitive impairment in English and Spanish versions. All scores were statistically adjusted for the effects of age, sex, education, and language of test administration. Participants with adjusted scores falling below the 10th percentile of the normative sample were considered impaired. The Informant Questionnaire on Cognitive Decline in the Elderly (IQCODE), which has been described extensively elsewhere, ${ }^{16-18}$ was also used for all participants undergoing SENAS testing. Participants were referred for a neurological examination if they met at least one of the following criteria: (1) a score below the 10th percentile on one or more of the six neuropsychological tests and a score on the IQCODE of 3.40 or greater (approximately $20 \%$ of the overall sample had scores $\geq 3.40$ ), (2) impaired scores on four or more neuropsychological tests regardless of IQCODE, or (3) IQCODE greater than 4.0 (about $5 \%$ of the overall sample had scores in this range). Forty-seven of the 62 participants with proxy interviews were referred for neuropsychological testing. A neurologist conducted the examination in the participants' homes assisted by a nurse or medical assistant.

A team of neurologists and a neuropsychologist adjudicated all potential dementia cases for a clinical diagnosis using criteria that required (1) clinically significant impairment in two or more separate cognitive domains that included a decline from premorbid function and (2) clinically significant impairment of independent functioning. Memory impairment was not required for a diagnosis of dementia ${ }^{19}$ as long as there was impairment in two other domains, but only five of the 69 fully evaluated cases did not have memory impairment on the SEVLT or the three-word recall of the 3MSE. Memory impairment was omitted as a criterion to bring the diagnostic categorization into conformity with the Alzheimer's Disease Diagnostic and Treatment Center (ADDTC) criteria for vascular dementia ${ }^{20}$ that also do not require memory loss per se, partly because antegrade amnesia, although an almost universal feature of Alzheimer's disease (AD), is not necessarily a feature of vascular dementia. Furthermore, dementia was diagnosed on clinical grounds so that the few patients who met criteria without memory loss had a progressive loss of at least two other cognitive functions from premorbid levels and functional impairment, clearly criteria that most clinicians would accept as indicative of dementia. Because memory and learning impairment are consistently associated with a diagnosis of $\mathrm{AD}$, all cases classified as $\mathrm{AD}$ met memory impairment criteria. However, all of the five cases without memory loss were classified as vascular or mixed dementia. Diagnoses were established on the basis of neuropsy- chological test scores and IQCODE scores but also included the history, mental status examination, and findings from the neurological examination when available. All cases were classified as normal, cognitively impaired but not demented (CIND), or demented. Demented cases were referred for magnetic resonance imaging (MRI) and appropriate laboratory tests. California ADDTC criteria ${ }^{20}$ were used to diagnose ischemic vascular dementia and $\mathrm{Na}$ tional Institute of Neurological Disorders and Stroke/ Alzheimers Disease and Related Disorders Association criteria were used to diagnose AD..$^{21,22}$

Of the 69 SALSA participants fully evaluated for dementia at baseline, five $(7.2 \%)$ did not have memory impairment on a neuropsychological test. All of these five cases had a history of stroke, diabetes mellitus, atrial fibrillation, or congestive heart failure at baseline. All had evidence of functional impairment related to cognitive decline (IQCODE $\geq 3.4$ ). All had two or more areas of low scores on other cognitive tests in the impaired range as follows: five failed each of the object naming test and the nonverbal semantic memory test, and four failed each of the verbal conceptualization, verbal attention, and the pattern recognition tests. One additional case was severely aphasic, and test data were available only on the 3MSE (score $=48$ / $100)$ and the object naming test (score $=2 / 20$ ).

ApoE genotyping was done using buccal cell DNA. After brushing the cheek mucosal surfaces, brushes were placed in 600 microliters of $50 \mathrm{mM}$ sodium hydroxide and heated at $950^{\circ} \mathrm{C}$ for 10 minutes. The method used followed a modification of polymerase chain reaction amplification/ Hhal restriction isotyping method. This includes a mutagenic forward primer and 5' end labeling of both primers. The initial method used for typing was a modification of Appel et al., ${ }^{23}$ except that the laboratory used fluor conjugated forward and reverse primers to allow detection and electrophoretograms on a sequencer. The sequence surrounding the single nucleotide polymorphisms (SNPs) matched precisely the published sequences.

\section{Statistical Approach}

These analyses include only participants who had at least one screening test at baseline. Eleven people were excluded by these criteria. Bivariate comparisons of participants' characteristics were made using frequencies and chi-square tests for differences. Continuous variables were compared using a linear regression model or an analysis of variance (SAS general linear model (SAS Institute, Inc., Cary, NC)). Sensitivity, specificity, and positive and negative predictive values of the screening criteria in identifying dementia were calculated using a positive value on either of the screening tests and based on formulae published by Sackett. ${ }^{24}$ Participants with a diagnosis of dementia were coded as 1 , and those without a diagnosis were assigned to the 0 category. One hundred thirty-two people of $1,779(7.4 \%)$ who were screened met screening criteria for referral but did not progress to neuropsychological testing because of death or refusal. The probability of dementia was estimated by imputing diagnosis from a weighted polytomous logistic regression model that excluded cases that were not fully evaluated. Covariates in the model included sex, age, 3MSE and DelRec, tagged status, and education; the dependent variable was cognitive status (demented, CIND, or normal). 
The reciprocal weights for the model were estimated based on tagged status and impaired status. The model estimated an individual probability for each case. For initially impaired cases without a full evaluation, both a moderate $(\geq 85$ th percentile) and a high probability ( $\geq 90$ th percentile) were assigned. All fully evaluated cases received their original classification (normal, CIND, demented). This is similar to an approach employed by Hendrie et al. ${ }^{25}$ Prevalence numbers reported here combine fully diagnosed cases with those determined by this estimation method. Confidence limits for the age-specific and overall prevalence estimates were derived using formulae provided by Fleiss. ${ }^{26}$ For examining sex differences in dementia risk by education, education was coded as none versus any. Median education in those with at least 1 year of education was 8 years. Risk of dementia associated with key risk factors was estimated using a logistic regression model that included age and other covariates as noted. A chi-square test was used to test whether the sample was in Hardy-Weinberg equilibrium for the ApoE gene.

\section{RESULTS}

Overall, the distribution by age group and sex is similar for the SALSA sample and the 1998 Census Dress Rehearsal data for Sacramento County. ${ }^{7}$ Tables 1 and 2 show the sample characteristics for all SALSA participants. Almost half $(49.0 \%)$ of the participants were born in the United States, $45.4 \%$ in Mexico, and $5.6 \%$ in another Latin American country. Spanish was the language of interview for nearly $58 \%$ of the sample. Nearly $89 \%$ of those born in Mexico or Latin America spoke primarily Spanish. Education among English speakers was significantly higher than among Spanish speakers. Thirteen percent $(13.4 \%)$ of the sample had no formal education. Women had significantly less education than men (mean \pm standard error $(\mathrm{SE})=$ $6.93 \pm 0.16$ years vs $7.7 \pm 0.19$ years, $P=.004)$.

Table 1. Cultural and Sociodemographic Sample Characteristics in the Sacramento Area Latino Study on Aging

\begin{tabular}{lccl}
\hline & \multicolumn{2}{c}{ Primary Language } & \\
\cline { 2 - 3 } \multicolumn{1}{c}{ Characteristic } & English & Spanish & \multirow{2}{*}{ Total } \\
\hline Country of birth, $\mathrm{n}(\%)$ & & & \\
$\quad$ Mexico & $91(12.2)$ & $716(69.6)$ & $807(45.4)$ \\
Other Latin & & & \\
$\quad$ American country & $12(1.6)$ & $88(8.6)$ & $100(5.6)$ \\
United States & $646(86.2)$ & $225(21.9)$ & $871(49.0)$ \\
Total & $749(42.1)$ & $1,029(57.9)$ & \\
Education, years & & & \\
None, $\mathrm{n}(\%)$ & $16(2.14)$ & $222(21.6)$ & $238(13.4)$ \\
$1-8, \mathrm{n}(\%)$ & $216(28.8)$ & $629(61.1)$ & $845(47.5)$ \\
$9-11, \mathrm{n}(\%)$ & $111(14.8)$ & $65(6.3)$ & $176(9.9)$ \\
$12, \mathrm{n}(\%)$ & $172(22.96)$ & $53(5.2)$ & $225(12.7)$ \\
$>12, \mathrm{n}(\%)$ & $234(31.2)$ & $60(5.8)$ & $294(16.5)$ \\
Mean \pm standard & & & \\
error & $10.76 \pm 4.4$ & $4.69 \pm 4.4$ & \\
Median & 12.0 & 3.0 & \\
Range & $0-32$ & $0-24$ & \\
\hline
\end{tabular}

Table 2. Age of Men and Women in the Sacramento Area Latino Study on Aging

\begin{tabular}{lccc}
\hline & Men & Women & Total \\
\cline { 2 - 4 } \multicolumn{1}{c}{ Age } & \multicolumn{3}{c}{$\mathrm{n}(\%)$} \\
\hline $60-69$ & $381(51.5)$ & $488(47.01)$ & $869(48.8)$ \\
$70-79$ & $279(37.7)$ & $425(40.94)$ & $704(39.6)$ \\
$80-84$ & $50(6.76)$ & $74(7.13)$ & $124(7.0)$ \\
$85+$ & $30(4.05)$ & $51(4.91)$ & $81(4.6)$ \\
Total & $740(41.6)$ & $1,038(58.4)$ & 1,778 \\
Mean \pm standard & & & \\
$\quad$ error & $70.5 \pm 6.96$ & $70.7 \pm 7.24$ \\
Median & 69.0 & 70.0 \\
Range & $60-101$ & $60-98$ \\
\hline
\end{tabular}

Figure 1 shows the number of participants who completed the steps for dementia ascertainment. Nearly $27 \%$ $(\mathrm{n}=476)$ met screening criteria for further neuropsychological testing. Of these, 123 met criteria for dementia on the neuropsychological tests and underwent a clinical examination. Of those examined, 118 met criteria for dementia or CIND.

\section{Prevalence by Age and Sex}

Table 3 shows the prevalence of dementia for fully evaluated cases and all cases, along with $95 \%$ confidence intervals (CIs) for each estimate. There were 69 fully diagnosed cases of dementia. The probability estimation procedure added 16 cases. Overall dementia prevalence was 3.8\% for the fully evaluated estimate. Estimates were $4.8 \%$ for women and $4.7 \%$ for men. Estimates for dementia prevalence by age for women aged 85 and older were $17.7 \%$ to $37.3 \%$. Estimates for men aged 85 and older ranged from
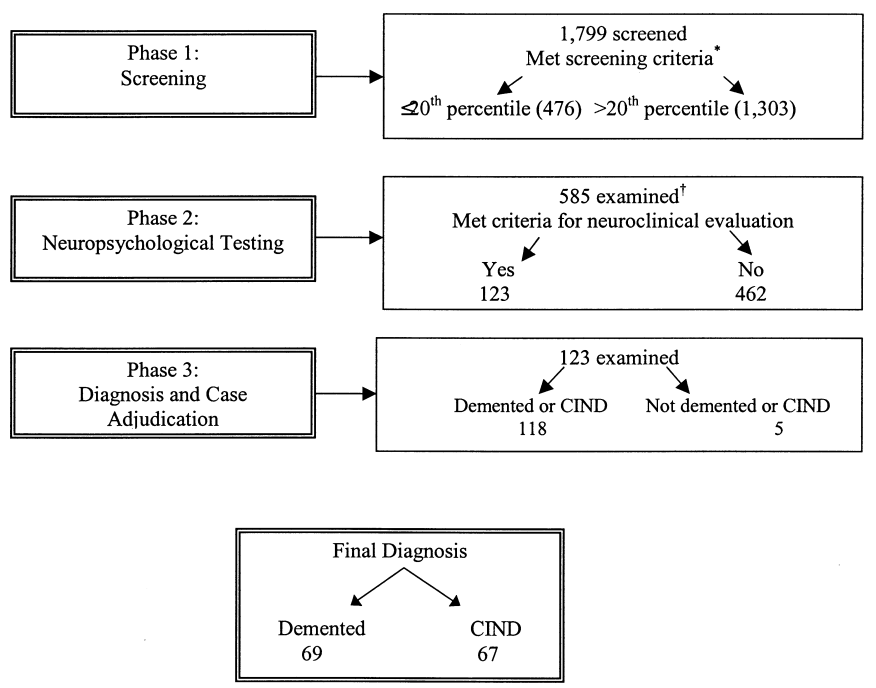

Figure 1. Steps for dementia ascertainment. "Age- and education-adjusted scores. ${ }^{\dagger}$ Including those meeting screening criteria and the randomly selected subsample. Eleven refused cognitive screening at baseline. CIND = cognitively impaired but not demented. 
Table 3. Prevalence of Dementia by Age and Gender for Fully Evaluated and All Cases

\begin{tabular}{ccc}
\hline & \multicolumn{1}{c}{$\begin{array}{c}\text { Fully } \\
\text { Evaluated } \\
\text { Cases }\end{array}$} & All Cases \\
\cline { 2 - 3 } & \multicolumn{2}{c}{$\mathrm{n}(\%)$} \\
Age & \multicolumn{2}{c}{$(95 \%$ Confidence Interval) } \\
\hline Women & \multicolumn{3}{c}{$3(0.61)$} \\
$60-69$ & $3(0.61)$ & $(0.37-1.06)$ \\
& $(0.37-1.06)$ & $2(4.71)$ \\
$70-79$ & $20(4.71)$ & $(3.8-5.8)$ \\
& $(3.8-5.8)$ & $8(10.81)$ \\
$80-84$ & $7(9.46)$ & $(7.9-15.0)$ \\
& $(6.8-13.5)$ & $19(37.25)$ \\
$\geq 85$ & $9(17.65)$ & $(31.6-44.9)$ \\
& $(13.4-23.9)$ & $50(4.82)$ \\
All & $39(3.76)$ & $(4.2-5.5)$ \\
& $(3.2-4.4)$ & \\
Men & & $5(1.31)$ \\
$60-69$ & $5(1.31)$ & $(0.9-2.0)$ \\
& $(0.9-2.0)$ & $17(6.09)$ \\
$70-79$ & $13(4.66)$ & $(4.9-7.7)$ \\
& $(3.6-6.1)$ & $6(12.00)$ \\
$80-84$ & $5(10.0)$ & $(8.5-17.5)$ \\
& $(6.8-15.2)$ & $7(23.33)$ \\
$\geq 85$ & $7(23.33)$ & $(17.4-32.5)$ \\
& $(17.4-32.5)$ & $35(4.72)$ \\
All & $30(4.05)$ & $(4.0-5.6)$ \\
& $(3.4-4.8)$ & \\
\hline
\end{tabular}

$23 \%$ to nearly $27 \%$. Age was significantly associated with an increased risk of dementia (odds ratio (OR) (for a 1year difference) $=1.18,95 \% \mathrm{CI}=1.15-1.22)$. There was no difference between male $(\mathrm{M})$ and female $(\mathrm{F})$ in risk of dementia $^{1}(\mathrm{OR}(\mathrm{M} / \mathrm{F})=0.95,95 \% \mathrm{CI}=0.62-1.47)$. However, education (none vs any) modified the association between sex and dementia. In those with no education, the risk of dementia was nearly four times higher in men than in women $(\mathrm{OR}(\mathrm{M} / \mathrm{F})=3.61,95 \% \mathrm{CI}=1.25$ $10.42)$. In those with any education, there was no sex difference in dementia risk $(\mathrm{OR}=0.75,95 \% \mathrm{CI}=0.40$ 1.38). Education (in years) was negatively associated with dementia $^{1}$ (OR $(1$-year difference) $=0.94,95 \% \mathrm{CI}=0.89$ $0.98)$. Cultural orientation and education years were correlated at 0.56 (Spearman rank correlation). The influence of cultural orientation on dementia risk was also modified by sex and education. In women with no education, Mexican cultural orientation was not associated with dementia but was associated with an increased risk of dementia in men $(\mathrm{OR}$ (1-unit change in cultural score) $=1.11,95 \%$ $\mathrm{CI}=1.03-1.20)$. In those with any education ( $\geq 1$ year), there was no association between cultural orientation and dementia risk for either sex.

\section{Accuracy of the Screening Tests}

Sensitivity, specificity, and positive predictive values were examined by tagged status (see Table 4). There were no differences in the sensitivity of specificity of the screening
Table 4. Sensitivity and Specificity of the Screening Criteria for Identifying Dementia for Those Meeting Criteria and a Randomly Selected Subsample of Entire Population

\begin{tabular}{|c|c|c|c|}
\hline \multirow[b]{2}{*}{ Screening Test Result } & Demented & $\begin{array}{c}\text { Not } \\
\text { Demented }\end{array}$ & Total \\
\hline & \multicolumn{3}{|c|}{$\mathrm{n}$} \\
\hline \multicolumn{4}{|l|}{$\begin{array}{l}\text { Met screening criteria } \\
\quad(\text { untagged })^{\star}\end{array}$} \\
\hline Impaired & 51 & 321 & 372 \\
\hline Normal & 0 & 1,044 & 1,044 \\
\hline Total & 51 & 1,365 & 1,416 \\
\hline \multicolumn{4}{|l|}{$\begin{array}{l}\text { Random subsample } \\
{\text { (tagged })^{\dagger}}^{\text {(tage }}\end{array}$} \\
\hline Impaired & 18 & 86 & 104 \\
\hline Normal & 0 & 259 & 259 \\
\hline Total & 18 & 345 & 363 \\
\hline
\end{tabular}

${ }^{*}$ Efficacy of screening tests: sensitivity $=100 \%$, specificity $=76.5 \%$, predictive value $=13.7 \%$

${ }^{\dagger}$ Efficacy of screening tests: sensitivity $=100 \%$, specificity $=75.1 \%$, predictive value $=17.3 \%$

criteria for detecting dementia whether the sample was selected randomly or met screening criteria. The prevalence of dementia in fully evaluated cases was $3.6 \%$ in untagged and $5.0 \%$ in tagged cases. This was not a statistically significant difference $(P=.23)$. In short, sensitivity of the screening tests was similar whether the participant was randomly selected from the entire sample or referred on the basis of cognitive screening scores. Random selection of cases for further evaluation (tagged) did not affect the accuracy of the screening tests in identifying cases or noncases.

\section{Cognitive Screening Scores and Functional Status by Dementia}

Table 5 shows the means and SEs by dementia classification for the 3MSE and the word list (DelRec) for the ageand education-adjusted cognitive screening test scores. Also shown are means and SEs for ADLs, IADLs, age, cultural orientation, and education. All cognitive scores, functional status, education, and mean age for dementia by any criteria were lower than for those not demented. Those who were fully diagnosed had slightly more education than those who were classified by probability scores. Cultural orientation did not differ within dementia groups but was higher within the nondemented than the demented groups.

\section{Etiology}

Table 6 shows the etiology of those classified as demented and the classification of cognitively impaired cases, deaths, and cases for whom etiology could not be determined. Approximately $55 \%$ of those cases for which an etiology could be determined $(\mathrm{n}=48)$ were classified as $\mathrm{AD}, 31 \%$ were vascular dementia, and nearly $4 \%$ were mixed.

\section{Type 2 Diabetes Mellitus and Stroke}

The prevalence of type 2 diabetes mellitus and stroke in this sample was $32.7 \%(\mathrm{n}=585)$ and $9.5 \%(\mathrm{n}=168)$, 
Table 5. Means for Cognitive Screening Test Scores, Age, Cultural Orientation, and Education in Years by Diagnosis of Dementia from a Regression Model

\begin{tabular}{lrrr}
\hline & \multicolumn{1}{c}{$\begin{array}{c}\text { Fully } \\
\text { Evaluated }\end{array}$} & $\begin{array}{c}\text { All } \\
\text { Cases }^{\dagger}\end{array}$ \\
\cline { 2 - 4 } \multicolumn{1}{c}{ Covariate (Range) } & Nondemented & \multicolumn{2}{c}{ Mean \pm Standard Error } \\
\cline { 2 - 4 } & & $48.1 \pm 1.51$ & $47.3 \pm 1.29$ \\
Modified Mini-Mental State Examination (0-100) & $85.6 \pm 0.30$ & $2.4 \pm 0.36$ & $2.4 \pm 0.31$ \\
Delayed Word List Recall (0-15) & $8.55 \pm 0.07$ & $3.86 \pm 0.38$ & $4.3 \pm 0.33$ \\
Activities of daily living (0-21) & $0.67 \pm 0.06$ & $14.36 \pm 1.12$ & $15.8 \pm 0.96$ \\
Instrumental activities of daily living (0-45) & $5.88 \pm 0.17$ & $79.0 \pm 0.83$ & $80.2 \pm 0.74$ \\
Age (60-101) & $70.3 \pm 0.17$ & $16.7 \pm 0.78$ & $15.9 \pm 0.70$ \\
Cultural orientation (0-37) & $17.8 \pm 0.16$ & $5.4 \pm 0.64$ & $4.9 \pm 0.56$ \\
Education (0-32) & $7.3 \pm 0.13$ & & \\
\hline
\end{tabular}

${ }^{\star} P<.05$ compared with diagnosed.

$\dagger$ Includes fully diagnosed and imputed cases.

respectively. The risk of dementia for patients with stroke combined with type 2 diabetes mellitus was more than eight times higher for fully evaluated cases and more than seven times higher for all cases than for those without diabetes and stroke. Forty percent of the dementia cases had type 2 diabetes mellitus, stroke, or a combination. Twentysix percent $(\mathrm{n}=9)$ of $\mathrm{AD}$ cases had type 2 diabetes mellitus, $5.9 \%(\mathrm{n}=2)$ had stroke, and $11.8 \%(\mathrm{n}=4)$ had both; $6.25 \%(\mathrm{n}=1)$ percent of vascular dementia cases had type 2 diabetes mellitus, $12.5 \%(\mathrm{n}=2)$ had stroke, and $62.5 \%(\mathrm{n}=10)$ had both; $16.7 \%(\mathrm{n}=1)$ percent of mixed cases had type 2 diabetes mellitus, $33.3 \%(n=2)$ had stroke, and $50 \%(\mathrm{n}=3)$ had both.

\section{ApoE Genotyping}

Eighty-five percent $(\mathrm{n}=1,614)$ of the cohort was genotyped for ApoE lipoprotein (175 refused to have buccal

Table 6. Etiology of Dementias and Classification of Cognitively Impaired or Normal Cases in the Sacramento Area Latino Study on Aging Baseline Sample ( $N=1,779)$

\begin{tabular}{lc}
\hline \multicolumn{1}{c}{ Etiology or Diagnosis } & $\mathrm{n}(\%)$ \\
\hline All demented cases & \\
Not demented & $1,651(92.9)$ \\
Possible AD & $10(13.0)$ \\
Probable AD & $21(30.4)$ \\
Possible VD & $2(2.9)$ \\
Probable VD & $12(17.4)$ \\
Mixed AD and VD & $3(4.3)$ \\
Other dementia & $3(4.3)$ \\
Possible Lewy Body dementia & $1(1.4)$ \\
Dementias with undetermined etiology & $17(18.8)$ \\
Total dementias & $69(100.0)$ \\
Total screened sample & \\
No clinical examination, no etiology & $1(0.05)$ \\
Cognitively impaired but not demented & $57(3.2)$ \\
Deaths & $65(3.6)$ \\
\hline
\end{tabular}

Note: Participants $(\mathrm{n}=11)$ that did not have at least one screening test were excluded from these analyses.

$\mathrm{AD}=$ Alzheimer's disease; $\mathrm{VD}=$ vascular dementia swabs taken) (Table 7). Allele frequencies were as follows: $\mathrm{E} 2=5.9 \%, \mathrm{E} 3=90.1 \%$, and $\mathrm{E} 4=4.0 \%$. The percentage distribution for each allelic combination was $2-2(0.93 \%)$, 2-3 (9.6\%), 2-4 (0.50\%), 3-3 (82.4\%), 3-4 (5.9\%), and 4-4 $(0.81 \%)$. Allele combinations were recoded into four indicator variables to allow the comparison of those heterozygous or homozygous for alleles including 2 or 4 to the $3-3$ combination. This was done using the following coding: Those with one 2 allele were coded as present $(1=2$ 3 or $2-4)$ on an indicator variable compared with those with a double 2 allele or no 2 allele (0). Those with a double 2 allele were coded as present $(1=2-2)$ on another indicator variable compared with those with a single 2 allele or no 2 allele (0). An identical procedure created two indicator variables for the presence or absence of a single (2-4 or 3-4) or a double 4 (4-4) allele. In addition, to be consistent with other studies, those with any 4 allele present $(2-4,3-4$, or $4-4)$ were also categorized and analyzed separately. The odds ratios shown are compared with the 3-3 group. The risk of dementia was highest in the group homozygous for the ApoE4 allele. The risk ratios of those homozygous for 2 and those heterozygous for 4 were of the same magnitude as the 3-3 group. All of the CIs for these estimates included 1.0, because of the small number of dementia cases in most of the cells.

\section{DISCUSSION}

The SALSA dementia prevalence estimates are lower than those reported for Caribbean Latinos in New York City ${ }^{3}$ but similar to those reported for the European Studies of Dementia studies ${ }^{27}$ and the Canadian Study on Aging. ${ }^{28}$ Given that Caribbean Latinos and Mexican Americans are historically, culturally, and ethnically different, this finding is not surprising. Some of these differences may be accounted for by differences in screening tests and diagnostic algorithms and limitations on the ability to completely evaluate every referred participant for dementia. In this study, the dementia prevalence estimates, especially after accounting for cases that were not fully evaluated, are similar to other studies. The age and education adjustment procedures did not substantially affect the overall sensitivity of the screening tests and improved the specificity. Other work has suggested that adjustment for education 
Table 7. Association Between Apolipoprotein E Genotype, Dementia Prevalence, and Cognitive Impairment in the Sacramento Area Latino Study on Aging

Allele Combination

\begin{tabular}{|c|c|c|c|c|c|c|}
\hline Dementia Status & $2-2$ & $2-3$ or $2-4$ & $3-3$ & $2-4$ or $3-4$ & $4-4$ & Any E4+ \\
\hline $\begin{array}{l}\text { Subjects with dementia, } \\
\mathrm{n}(\%)\end{array}$ & $15(1.27)$ & $162(11.4)$ & $1,327(4.85)$ & $101(6.4)$ & $13(2.5)$ & $114(5.26)$ \\
\hline $\begin{array}{l}\text { Risk of dementia, risk ratio } \\
(95 \% \text { confidence interval) } \\
\text { Modified Mini-Mental State } \\
\text { Examination score, } \\
\text { mean } \pm \text { standard error }{ }^{\dagger}\end{array}$ & $1.02(0.46-2.28)$ & $1.38(0.47-4.09)$ & 1.0 (Ref) & $1.02(0.35-3.02)$ & $2.04(0.88-4.72)$ & $1.37(0.55-3.37)$ \\
\hline $\begin{array}{l}\text { Demented } \\
\text { Nondemented }\end{array}$ & $\begin{array}{l}65.1 \pm 12.8 \\
85.05 \pm 3.6\end{array}$ & $\begin{array}{r}52.75 \pm 8.6 \\
84.9 \pm 2.6\end{array}$ & $\begin{aligned} 47.86 & \pm 1.44 \\
86.4 & \pm 0.33\end{aligned}$ & $\begin{array}{l}51.3 \pm 9.4 \\
86.3 \pm 2.7\end{array}$ & $\begin{array}{l}56.9 \pm 10.7 \\
85.2 \pm 3.9\end{array}$ & $\begin{array}{l}43.8 \pm 4.7 \\
876+11\end{array}$ \\
\hline
\end{tabular}

* From a logistic regression model including age and gender, where 3-3 is the reference category.

${ }^{\dagger}$ From an analysis of variance including age, gender, dementia status, and the four gene combinations.

and age may not significantly affect the sensitivity or specificity of cognitive screening tests ${ }^{29,30}$ and may influence the estimates of dementia risk associated with these factors. Thus, such adjustment may not be necessary to achieve an accurate estimate of dementia prevalence.

The SALSA sample appears to be representative of the target population from which it was drawn. The age and sex distributions for this sample are similar to those of Sacramento County. The authors did not have access to data on education, income, or other covariates for the 1998 or 2000 Census for older Mexican Americans in this area, and making a comparison using 1990 Census data is not useful, because of its age. According to the Centers for Disease Control and Prevention, ${ }^{31}$ approximately 32,100 Mexican Americans aged 65 and older lived in nursing homes in 1997, or about $1.1 \%$ of all older Mexican Americans, compared with $4 \%$ of older white non-Latino people. Magaziner et al. ${ }^{32}$ has estimated that $30 \%$ of all dementia patients live in nursing homes, suggesting that studies of community-dwelling older people would tend to underestimate dementia prevalence. Given that the percentage of older Mexican Americans in nursing homes is smaller than that of non-Latino whites, ${ }^{31}$ the authors' prevalence estimates are probably closer to the true value than would be the case for studies of community-dwelling Anglos.

The frequency distribution of the ApoE genotype in this sample is similar to that reported in several other studies. ${ }^{33,34}$ Reports of ApoE frequency distributions in Mexican-American, Mexican mestizo, and Mayan populations have revealed an ApoE distribution characterized by a high frequency of E3, ranging from $89 \%$ to $92 \%$; E4 frequencies reported in these studies range from $6.9 \%$ to $8.4 \%$, and $\mathrm{E} 2$ frequencies tend to be low or absent in these ethnic groups. The E4 frequency in this study's sample is slightly lower than other reports. However, this sample is older than those of other studies for which ApoE genotype in Mexicans is reported. The frequency of ApoE4 tends to decline with age, as a function of differential population selection, and this might account for the lower frequency of E4. Those who are homozygotic for E4 appear to have higher dementia risk and lower cognitive scores.
Other studies have suggested an interaction between type 2 diabetes mellitus or stroke and ApoE4 such that the presence of both increases the risk of dementia or cognitive decline. ${ }^{35,36}$ Because type 2 diabetes mellitus prevalence is high in this sample and tends to have an early onset in Mexican Americans, the early selective effects of ApoE4, type 2 diabetes mellitus, or both may account for the lower E4 frequency as well. The ApoE4 distribution that this study found is similar to reports from several other studies of Mexican Indians ${ }^{33,34}$ and Mexican Americans. ${ }^{37-39}$ These reports have suggested that ApoE4 is associated with higher low-density lipoprotein levels and potentially with adverse metabolic changes related to type 2 diabetes mellitus.

These findings have important implications for future projections of dementia prevalence in the general population of older Mexican Americans. Type 2 diabetes mellitus is dramatically higher in Mexican Americans than in most other Latino groups and in Anglos. ${ }^{40}$ Overall prevalence of type 2 diabetes mellitus in older Mexican Americans has been estimated in other studies at 35\%.40-42 These figures are similar to those found in SALSA. If type 2 diabetes mellitus and related stroke continue to increase in this population, the prevalence of dementia may also increase. Approximately $43 \%$ of the dementia cases in this study have type 2 diabetes mellitus, stroke, or both. Nationally, there are an estimated 2.2 million Mexican Americans aged 60 and older, ${ }^{43}$ of whom $35 \%$ are estimated to have type 2 diabetes mellitus. By the authors' estimates, approximately 28,000 of these may be at increased risk for dementia due to the combined burden of type 2 diabetes mellitus and stroke. At minimum, these findings suggest that type 2 diabetes mellitus screening and treatment programs targeting older Mexican Americans need to include evaluation of cognitive effects. Only a few of the cases in this study had previously been diagnosed with dementia. All were living in their homes at the time they were initially contacted, and most were newly diagnosed by this study. Because $90 \%$ of the sample had medical insurance and $88 \%$ had a regular doctor, one can reasonably assume that their healthcare source failed to identify their dementia.

Education is a major factor in this study as in other 
research. ${ }^{44-46}$ These results show a strong and negative association between education and dementia. Mexican cultural orientation is also associated with a higher risk of dementia, although this appears to exist mainly in those with low education. There have been no other studies of cultural orientation in Mexican Americans in relation to dementia risk with which there results can be compared. Graves et al. ${ }^{47}$ reported a lower risk of cognitive impairment in Japanese Americans who remained oriented to Japanese culture, but there is no reason to believe that acculturation works in similar ways for all immigrant groups. The history of Mexican immigration differs ${ }^{48}$ substantially from that of Japanese immigration in several ways. Mexico is next to the United States, and the costs of immigration are lower, making economic and health status selection less of a factor. Lifestyle factors such as diet (possibly lower fat in traditional Japanese diets than Mexican) may differ. Socioeconomic status may be much lower in traditional Mexican Americans than in traditional Japanese. No sex differences were found in dementia risk except in those with no education, where men were at greater risk. The European Studies of Dementia study reported a stronger education effect for women than men with AD but not for vascular dementia. ${ }^{49} \mathrm{Sev}$ eral other studies have reported a higher risk of $\mathrm{AD}$ in women. ${ }^{45,50,51}$

This study is the first representative, population-based study of dementia in older Mexican Americans. The findings suggest that, although dementia prevalence in older Mexican Americans may not differ from that of Canadians and Europeans, the sources of dementia in this population may be different. The low frequency of ApoE4 and the small contribution to dementia presented by ApoE4, combined with the high prevalence of type 2 diabetes mellitus suggests that the etiology of dementia in Mexican Americans may be different from that of other populations.

\section{REFERENCES}

1. Graves AB, Larson EB, White LR et al. Opportunities and challenges in international collaborative epidemiologic research of dementia and its subtypes: Studies between Japan and the U.S. Int Psychogeriatr 1994;6:209-223.

2. Harwood DG, Barker WW, Loewenstein DA et al. A cross-ethnic analysis of risk factors for AD in white Hispanics and white non-Hispanics. Neurology 1999;52:551-556.

3. Gurland BJ, Wilder DE, Lantigua R et al. Rates of dementia in three ethnoracial groups. J Geriatr Psychiatry 1999;14:481-493.

4. Acevedo A, Loewenstein DA, Barker WW et al. Category fluency test: Normative data for English- and Spanish-speaking elderly. J Neuropsychol Soc 2000;6:760-769.

5. Arnold BR, Cuellar I, Guzman N. Statistical and clinical evaluation of the Mattis Dementia Rating Scale-Spanish adaptation: An initial investigation. J Gerontol B Psychol Sci Soc Sci 1998;53B:P364-P369.

6. Teresi JA, Golden RR, Cross P et al. Item bias in cognitive screening measures. Comparisons of elderly white, Afro-American, Hispanic and high and low education subgroups. J Clin Epidemiol 1995;48:473-483.

7. American Fact Finder [On-line]. Available at http://factfinder.census.gov Accessed October 2000.

8. Haan MN, González HM. Diversity is good science: The SALSA Study. Aging Health [In press.]

9. Cuellar I, Arnold B, Maldonado R. Acculturation Rating Scale for Mexican Americans. II. A revision of the original ARSMA Scale. Hispanic J Behav Sci 1995;17:274-304.

10. Katz SC, Ford AB, Moskowitz RW et al. Studies of illness in the aged: The index of ADL, a standardized measure of biological and psychosocial function. JAMA 1963;185:914-919.

11. Teng EL, Chui HC. The Modified Mini-Mental State (3MS) Examination. J Clin Psychiatry 1987;48:314-318.

12. González HM, Mungas DM, Reed BR et al. A new verbal learning and mem- ory test for English- and Spanish-speaking older people. J Int Neuropsychol Soc; 2001;7:544-555.

13. Mungas D, Marshall SC, Weldon M et al. Age and education correction of Mini-Mental State Examination for English and Spanish-speaking elderly. Neurology 1996;46:700-706.

14. Mungas D, Reed BR, Marshall SC. Development of psychometrically matched English and Spanish language neuropsychological tests for older persons. Neuropsychology 2000;14:209-223.

15. Mungas D. Psychometrically matched English and Spanish assessment scales for older persons. Arch Clin Neuropsychol 1999;14:712-713.

16. Jorm AF, Jacomb PA. Informant questionnaire on cognitive decline in the elderly (IQCODE): Socio-demographic correlates, reliability, validity and some norms. Psychol Med 1989;19:1015-1022.

17. Jorm AF, Scott R, Cullen JS et al. Performance of the informant questionnaires on cognitive decline in the elderly (IQCODE) as a screening test for dementia. Psychol Med 1991;21:785-790.

18. Morales JM. The screening of mild dementia with a shortened Spanish version of the IQCODE. Alzheimer Dis Assoc Dis 1995;9:105-111.

19. Knopman DS, DeKosky ST, Cummings JL et al. Practice parameters: Diagnosis of dementia (an evidence-based review). Report of the Quality Standards Subcommittee of the American Academy of Neurology. Neurology 2002;56:1143-1153.

20. Chui HC, Victoroff JJ, Margolin DI et al. Criteria for the diagnosis of ischemic vascular dementia proposed by the State of California Alzheimer disease diagnostic and treatment centers. Neurology 1992;42: 473-480.

21. Tierney MC, Black SE, Szalai JP et al. Recognition memory and verbal fluency differentiate probable Alzheimer dementia from subcortical ischemic vascular dementia. Arch Neurol 2001;58:1654-1659.

22. McKhann G, Drachman D, Folstein M et al. Clinical diagnosis of Alzheimer's disease: Report of the NINCDS-ADRDA Work Group under the auspices of Department of Health and Human Services Task Force on Alzheimer's Disease. Neurology 1984;34:939-944.

23. Appel E, Eisenberg S, Roitelman J. Improved PCR amplification/Hhal restriction for unambiguous determination of apolipoprotein $\mathrm{E}$ alleles. Clin Chem 1995;41:187-190.

24. Sackett DL. Clinical Epidemiology: A Basic Science for Clinical Medicine. Boston: Little, Brown, 1991.

25. Hendrie HC, Ogunniyi A, Hall KS et al. Incidence of dementia and Alzheimer disease in 2 communities. JAMA 2001;285:739-747.

26. Fleiss JL. Statistical Methods for Rates and Proportions. New York: John Wiley \& Sons, 1981.

27. Lobo A, Launer LJ, Fratiglioni L et al. Prevalence of dementia and major subtypes in Europe: A collaborative study of population-based cohorts. Neurologic Diseases in the Elderly Research Group. Neurology 2000;54:S4-S9.

28. Canadian study of health and aging: Study methods and prevalence of dementia. Can Med Assoc J 1994;150:899-913.

29. Ganguli M, Belle S, Ratcliff G et al. Sensitivity and specificity for dementia of population-based criteria for cognitive impairment: The MoVIES project. J Gerontol 1993;48:M152-M161.

30. Kraemer HC. Adjusting Mini Mental State Examination scores for age and educational level to screen for dementia: Correcting bias or reducing validity? Int Psychogeriatr 1998;10:43-51.

31. Centers for Disease Control and Prevention. Advance Data, April 2000 [Online]. Available at http://www.cdc.gov/nchs/about/major/nnhsd/nnhslist.htm Accessed October 30, 2002.

32. Magaziner J, German P, Zimmerman SI et al. The prevalence of dementia in a statewide sample of new nursing home admissions aged 65 and older: Diagnosis by expert panel. Epidemiology of Dementia in Nursing Homes Research Group. Gerontologist 2000;40:663-672.

33. Gamboa R, Hernandez-Pacheco G, Hesiquio R et al. Apolipoprotein E polymorphism in the Indian and Mestizo populations of Mexico. Hum Biol 2000 72:975-981.

34. Kamboh MI, Crawford MH, Aston CE et al. Population distributions of APOE, APOH, and APOA4 polymorphisms and their relationships with quantitative plasma lipid levels among the Evenki herders of Siberia. Hum Biol 1996;68:231-243.

35. Haan MN, Shemanski L, Jagust WJ et al. The role of APOE epsilon4 in modulating effects of other risk factors for cognitive decline in elderly persons. JAMA 1999;282:40-46.

36. Slooter AJ, Tang MX, van Duijn CM et al. Apolipoprotein E epsilon4 and the risk of dementia with stroke. A population-based investigation. JAMA 1997;277:818-821.

37. Haffner SM, Stern MP, Miettinen H et al. Apolipoprotein E polymorphism and LDL size in a biethnic population. Arterioscler Thromb Vasc Biol 1996; 16:1184-1188.

38. Shriver MD, Boerwinkle E, Hewett-Emmett D et al. Frequency and effects of 
apolipoprotein E polymorphism in Mexican-American NIDDM subjects. Diabetes 1991;40:334-337.

39. Valdez R, Howard BV, Stern MP et al. Apolipoprotein E polymorphism and insulin levels in a biethnic population. Diabetes Care 1995;18:992-1000.

40. Harris MI. Prevalence of diabetes, impaired fasting glucose, and impaired glucose tolerance in U.S. adults: The Third National Health and Nutrition Examination Survey (NHANES), 1988-94. Diabetes Care 1998;21:518-524.

41. Flegal KM. Prevalence of diabetes in Mexican Americans, Cubans, and Puerto Ricans from the Hispanic Health and Examination Survey (HHANES), 1982-84. Diabetes Care 1991;14:628-638.

42. Flegal KM, Ezzati TM, Harris MI et al. Prevalence of diabetes in Mexican Americans, Cubans, and Puerto Ricans from the Hispanic Health and Nutrition Examination Survey, 1982-84. Diabetes 1991;14:628-638.

43. U.S. Census Bureau. Population by age, Hispanic origin and race, and Sex: March, 1999 [On-line]. Current Population Survey Internet release, Available at http://www.census.gov/ Accessed March 8, 2000.

44. Kaplan GA, Turrell G, Lynch JW et al. Childhood socioeconomic position and cognitive function in adulthood. Int J Epidemiol 2001;30:256-263.

45. Launer LJ, Andersen K, Dewey ME et al. Rates and risk factors for dementia and Alzheimer's disease: Results from EURODEM pooled analyses. EURO-
DEM Incidence Research Group and Work Groups. European Studies of Dementia. Neurol 1999;52:78-84.

46. Lindsay J, Hebert R, Rockwood K. The Canadian Study of Health and Aging: Risk factors for vascular dementia. Stroke 1997;28:526-530.

47. Graves AB, Rajaram L, Bowen JD et al. Cognitive decline and Japanese culture in a cohort of older Japanese Americans in King County, WA: The Kame Project. J Gerontol B Psychol Sci Soc Sci 1999;54B:S154-S161.

48. Gutierrez DG. Migration, emergent ethnicity and the 'Third Space': The shifting politics of nationalism in greater Mexico. J Am History 1999;86: 481-517.

49. Copeland JR, McCracken CF, Dewey ME et al. Undifferentiated dementia, Alzheimer's disease and vascular dementia: Age and gender-related incidence in Liverpool. The MRC-ALPHA Study. Br J Psychiatry 1999;175: 433-438.

50. Fratiglioni L, Viitanen M, von Strauss E et al. Very old women at highest risk of dementia and Alzheimer's disease: Incidence data from the Kungsholmen Project, Stockholm. Neurology 1997;48:132-138.

51. Zhang MY, Katzman R, Salmon D et al. The prevalence of dementia and Alzheimer's disease in Shanghai, China. Impact of age, gender, and education. Ann Neurol 1990;27:428-437. 